\section{Mit immuntherapeutischen Strategien gegen CML}

So wie z. B. bei der akuten lymphatischen Leukämie versuchen Hämatoonkologen auch zur Bekämpfung der chronischen myeloischen Leukämie (CML) das körpereigene Immunsystem zu nutzen. Ein Weg führt dabei über Interferon (IFN) a. Interferone hätten pleiotrope Effekte, betonte Dominik Wolf, Bonn, unter anderem myelosuppressive, aber eben auch immunaktivierende Eigenschaften. Bei der CML habe gezeigt werden können, dass IFN auch einen Einfluss auf die „immunologische Sichtbarkeit" der Leukämiezellen haben. Inzwischen gebe es mehrere Studien, in denen IFN a mit Tyro-

sinkinaseinhibitoren (TKI) zur Therapie bei CML geprüft werde, erklärte Wolf auf der DGHO-Jahrestagung 2016. Ein Beispiel sei die NORDIC-CML-Studie mit dem TKI Dasatinib. Schließlich sei in vielen präsentierten Daten der deutschen CML-Studiengruppe gezeigt worden, dass durch solche Kombinationstherapien ein tiefes molekulares Ansprechen, also die starke Reduktion des BCR-ABL-Gens, möglich sei. Wie Wolf berichtete, hat IFN viele Einflüsse auf natürliche Killerzellen (NK-Zellen) und auf leukämieassoziierte T-Zellen, die gegen CML-Zellen aktiviert werden. Wolfs Angaben zufolge soll nun in der TIGER-Studie der deutschen CML-Studiengruppe dieser Zusammenhang prospektiv geprüft werden, nämlich was eine Erhaltungstherapie mit pegyliertem IFN a nach der Kombinationstherapie und dann vor allem nach dem Absetzen der Therapie für die Patienten bedeutet. Die Aufnahme von Patienten werde bald beendet sein. Die Studie hat drei Phasen: die optimierte Induktionsphase, die Kon- solidierungsphase und die Nachbeobachtung nach Beendigung der medikamentösen Therapie. Auch Checkpointhemmer werden inzwischen zur Therapie von CML-Patienten geprüft. Wolf berichtete, dass in einer Studie bei Patienten, die den TKI abgesetzt haben, die Wirksamkeit der Kombination des PD-1-Hemmers Nivolumab mit einer Single-Shot-Therapie des CTLA-4-Inhibitors Ipilimumab geprüft werde. Durch die Blockade mit den beiden Antikörpern soll das Immunsystem gegen die Leukämiezellen aktiviert werden.

Aber auch das angeborene Immunsystem mit NK-Zellen rückt in den Fokus der Hämatoonkologen. Wolf zufolge exprimieren NK-Zellen, analog zu PD-1-Rezeptoren auf aktivierten T-Zellen, Rezeptoren, die ebenfalls als Checkpointmoleküle zu werten sind, sogenannte KIR („killer cell immunoglobulinlike receptors"). Ersten australischen Studiendaten zufolge hätten CML-Patienten, denen dieser Rezeptor fehle, ein längeres ereignisfreies Überleben gehabt als Patienten, deren NK-Zellen den Rezeptor getragen hätten, so Wolf. Solche Beobachtungen führten nun zur Entwicklung von Therapiestrategien, bei denen KIR blockiert werde. Wolf bezeichnete sie als "Checkpoint-Antikörper für NK-Zellen". Ein Kandidat sei Lirilumab, der die KIR blockiere und so die angeborene Immunität aktiviere. Es gebe bereits erste klinische Studien dazu, unter anderem eine Phase-I-Studie mit CML-Patienten, in der die Kombination mit Nivolumab getestet werde. Solche gegen KIR gerichteten Antikörper seien sehr innovativ. Peter Leiner

\section{Liquid Biopsy: Vom Hype zurück in die Realität}

Wenn es nach Klaus Pantel, Hamburg ginge, würde der Begriff „Liquid Biopsy“ primär die Messung zirkulierender Tumorzellen (CTC) erfassen. Inzwischen wird er aber auch für die Detektion von zellfreier Tumor-DNA (cfDNA), zellfreier mitochondrialer Tumor-RNA (cfmiRNA) und Exosomen als vor allem bei Apoptose der Tumorzellen freigesetzte Biomarker verwendet. „Wir wollen aber immer auch wissen, was die lebenden Zellen machen", betonte Patel. Die ehrgeizigen Ziele der Liquid Biopsy sind das Screening und die Früherkennung von
Krebs, die Abschätzung des Metastasierungsrisikos, Stratifizierung und Monitoring der Therapie, die Identifizierung therapeutischer Zielstrukturen und Resistenzmechanismen und die Verbesserung des Verständnisses der Biologie der Metastasierung.

Die „Liquid Biopsy“ umfasst laut Pantel derzeit etwa 50 verschiedene Testmethoden, daher sind internationale Bemühungen um eine Standardisierung wichtig. Aktuell prüft ein europaweites Konsortium die blutbasierte Diagnostik auf Basis von CTC, ctDNA, cfmiRNA und Exosomen bei Lungen- und HER2positivem Brustkrebs.

\section{ctDNA-Test im Alltag angekommen}

Nach der Zulassung von Osimertinib zur Therapie des Lungenkarzinoms mit einer T790M-Mutation des EGFR (,epidermal growth factor receptor") nach Progress unter anderen EGFR-Inhibitoren ist die cfDNA-Messung in der Routine angekommen. Michael Hummel, Berlin, warnte aber vor Fallstricken. Wichtig sei die Verwendung von dafür geeigneten Röhrchen mit speziellen Zusatzstoffen. Sonst kann es in den Blutproben durch das Absterben von Zellen bis zur Analyse zu einer Überlagerung der eigentlich gesuchten zellfreien Tumor-DNA kommen. „Viel freie DNA ist immer ein ganz schlechtes Zeichen", betonte er. Im Labor sollte dann rasch das Plasma gewonnen und bei $-80^{\circ} \mathrm{C}$ eingefroren werden. Um sicherzugehen, dass überhaupt TumorDNA in der Probe vorhanden ist, empfiehlt Hummel, andere tumorspezifische Marker mitlaufen zu lassen.

\section{Ringversuch zeigt Anforderungen}

Ein Standard für die T790M-Detektion gibt es noch nicht. In einem Ringversuch der Qualitätssicherungs-Initiative $\mathrm{Pa}$ thologie (QUIP) zeigten sich in verschiedenen Laboren mit unterschiedlichen Methoden auch sehr verschieden hohe cfDNA-Mengen und T790M-Allelfrequenzen. Auch hier waren einige Proben durch Nichttumor-DNA kontaminiert, sodass die Detektion von T790M nicht möglich war. Letztlich nahmen 40 von 48 Laboren erfolgreich am Ringversuch teil. Eine Empfehlung für eine bestimmte Methode konnte nicht abgeleitet werden. Aber: „Es ist mindestens die digitale Polymerasekettenreaktion notwendig", betonte Hummel.

Friederike Klein

\section{Patientencoaching führt zu günstigerem Therapieverlauf}

„Patienten, die eine systematische Schulung von medizinischen Fachangestellten erhalten haben, haben ein besseres therapiebezogenes Wissen, neigen zu einer höheren Selbstwirksamkeit, haben weniger Nebenwirkungen wie Hautausschläge oder Schmerzen, brechen die Therapien seltener $a b$, haben weniger Fragen und entlasten den Arzt", erklärte Manfred Welslau, Aschaffenburg, mit Blick auf die Ergebnisse einer Interventionsstudie bei Patienten, die eine orale 